\title{
Harnessing Bacterial Power in Microscale Actuation
}

\author{
A. Agung Julius*, M. Selman Sakar*, Edward Steager ${ }^{\dagger}$, U Kei Cheang ${ }^{\dagger}$, \\ MinJun Kim ${ }^{\dagger}$, Vijay Kumar*, and George J Pappas*
}

\begin{abstract}
This paper presents a systematic analysis of the motion of microscale structures actuated by flagellated bacteria. We perform the study both experimentally and theoretically. We use a blotting procedure to attach flagellated bacteria to a buoyancy-neutral plate called a microbarge. The motion of the plate depends on the distribution of the cells on the plate and the stimuli from the environment.

We construct a stochastic mathematical model for the system, based on the assumption that the behavior of each bacterium is random and independent of that of its neighbors. The main finding of the paper is that the motion of the barge plus bacteria system is a function of a very small set of parameters. This reduced-dimensional model can be easily estimated using experimental data. We show that the simulation results obtained from the model show an excellent match with the experimentally-observed motion of the barge.
\end{abstract}

Keywords: microactuation, biological systems, flagellated bacteria.

\section{INTRODUCTION}

There has been a great deal of interest in micro robotics and micro manipulation with many applications, for example in microscale self assembly [1] and in robotic drug delivery and therapeutic mechanisms [2]. However, there are very few candidates for low- cost and reliable actuation, particularly since such micro robots must operate in a fluid. The idea of using microorganisms, particularly bacteria, to actuate microscale structures is very appealing. One apparent advantage of this approach is the possibility to produce microorganisms with very cheap cost relatively easily [3]. Inspired by bacterial locomotion, Bell et al [4] propose a swimming mechanism based for micro-robots using artificial flagella creating nano coils. We too are inspired by this mechanism but our approach is slightly different. Instead of using man made structures that are modeled after biological systems, we utilize actual microorganisms as microactuators.

The main challenges that need to be addressed in realizing the idea of using bacterial power to actuate microstructures are

1) how to fabricate the structures and integrate the bacteria to them, and

2) what is the behavior of the swarm of bacteria under certain environmental conditions and how to regulate it.

There have been a few pioneering contributions that address these challenges. For example, Martel et. al. used flagellated magnetotactic bacteria and demonstrated an accurate and predictive motion under computer control [5], Sitti

We gratefully acknowledge the support of ARO grant W911NF-05-10219 and NSF grants CSR-CPS 0720801 and CMMI-0745019.

*GRASP Laboratory, School of Engineering and Applied Sciences, University of Pennsylvania, Philadelphia, PA 19104, U.S.A. agung, sakarmah, kumar, pappasgegrasp. upenn.edu

${ }^{\dagger}$ Department of Mechanical Engineering and Mechanics, Drexel University, Philadelphia, PA 19104, U.S.A. esteager, ucheang, mkim@coe.drexel.edu

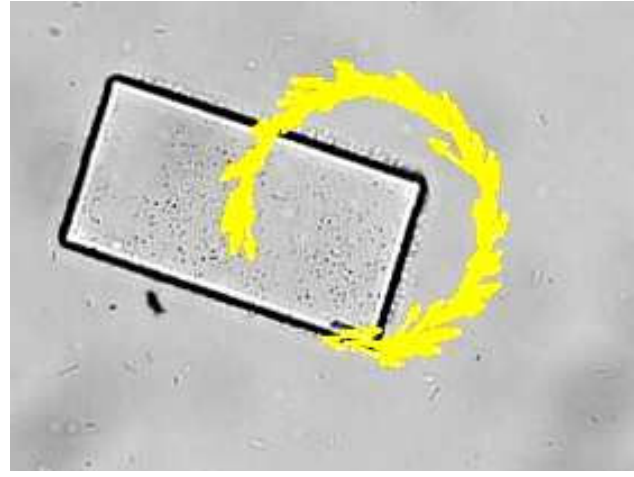

Fig. 1. A rectangular microbarge $(50 \mu \mathrm{m} \times 100 \mu \mathrm{m})$ that is used in this paper. The computer vision tracking system marks the trajectory of the microbarges and its computed interframe velocity with the arrows.

et. al. demonstrated on/off motion control using chemical stimuli [6], while Kim et. al. achieved similar results using ultraviolet light [7]. In this paper, we elucidate some microfabrication aspects of the challenge and quantitative mathematical modeling of the system.

We focus our attention to the chemotactic behavior of flagellated bacteria, such as Escherichia coli and Serratia marcescens. The motile behavior of these bacteria has been extensively studied since the 1970's (c.f. the seminal paper by Howard Berg [8], and a more recent book [9]). It has been established that these bacteria use their flagella to generate propulsion by rotating them [10] and that the motile behavior of the bacteria is similar to a biased random walk toward higher concentration of chemotactic attractant.

For this study, we build buoyancy-neutral plate-like microstructures, which we call microbarges. We then blot flagellated bacteria on the surface of the microbarge, which is then released to the medium. The motion of the microbarge is carefully tracked and compared with model prediction. This is shown in Figure 1.

We construct a stochastic mathematical model for the system, based on the assumption that the behavior of each bacterium is random and independent of that of its neighbors. In a recent paper [11], the authors proposed an approximate stochastic model to study the diffusion or random walk properties of microbeads with bacterial propulsion. In this paper, we study smooth and regular propulsion that is potentially more beneficial than random walk. The study of actuation by using a large number of random actuators has also been reported elsewhere, e.g. [12]. In addition to developing the stochastic model, we also perform parameter identification for the model, based on experimental data. We then demonstrate that the model with the estimated 
parameters is able to predict the behavior of the system very well. One of the key findings in this paper is that although the system is inherently distributed, in the sense that there are a large number of independent actuators ${ }^{1}$, we can construct an accurate model with only a few parameters representing the distribution of the bacteria.

\section{EXPERIMENTAL SETUP}

To accomplish effective actuation of custom designed microstructures several processes are necessary. These processes include culturing bacteria $S$. marcescens using the swarm plate technique, fabricating microstructures, blotting and manipulating microstructures with bacteria into the working fluid, and finally tracking the microstructures using an algorithm to quantify the magnitude and direction of motion.

\section{A. Cell Culturing}

Swarming $S$. marcescens were cultured on a $0.6 \%$ agar plate. To prepare agar plates for swarming, $5 \mathrm{~g}$ Difco Bacto tryptone, $2.5 \mathrm{~g}$ yeast extract, $2.5 \mathrm{~g} \mathrm{NaCl}$ and $3 \mathrm{~g}$ Difco Bacto agar are dissolved into $500 \mathrm{ml}$ of deionized water. After autoclaving the solution was poured into smaller bottles for later redistribution to Petri dishes. Before pouring individual agar plates, the agar solution was mixed with $25 \%$ glucose solution by adding $1 \mathrm{ml}$ glucose solution for $100 \mathrm{ml}$ of prepared agar solution. Then, $50 \mathrm{ml}$ of this new agar solution was pipetted into large $14 \mathrm{~cm}$ Petri dishes. The swarm plate was inoculated on one edge with $2 \mu \mathrm{l}$ of $S$. marcescens saturated culture. Agar plates were incubated at $30-34 \mathrm{C}$, and swarming began within 8-16 hours.

\section{B. Microfabrication}

The fabricated structures should be biocompatible, i.e. the structure material should preserve and promote bacterial motility and provide a surface to which bacteria can attach readily. Additionally, the composite specific gravity of the structure should be similar to the working fluid and provide both chemical and thermal stability. It is additionally helpful if the fabricated structures are transparent and have a high refractive index to provide clearly defined boundaries which can be readily discerned by a tracking algorithm.

1) Mask design: Masks are an integral component in the photolithographic process of microstructure fabrication. Using AutoCAD, the designed two-dimensional microgeometry was drawn with precision, and printed onto a transparency film (CAD/Art Service, Inc, Bandon, OR) with high resolution (18,000 dpi). A dark field mask design for microstructures was generated with $50 \times 100 \mu \mathrm{m}^{2}$ rectangles placed in an array. The distance between each individual pattern was approximately $40 \mu \mathrm{m}$ to allow working space for extraction of individual microstructures.

2) SU-8 microstructure fabrication: SU-8 Series 10 (MicroChem, Newton, MA) negative photoresist forms strong cross links on exposure to ultraviolet (UV) light, and the unexposed regions are easily removed using a developer solution. A two inch silicon wafer was first cleaned in isopropanol. The wafer was then dried with nitrogen gas,

\footnotetext{
${ }^{1}$ There is no known intercellular signalling component in the motile behavior of $E$. coli and $S$. marcescens.
}

rinsed with deionized water, and dehydrated at $200 \mathrm{C}$ for 5 minutes. Once the wafer was pre-treated, it was placed on the vacuum chuck of a spin coater. SU-8 10 negative photoresist was dispensed on the wafer to cover $2 / 3$ of the wafer surface or $1 \mathrm{ml}$ per inch of diameter. In order to achieve a final thickness of $10 \mu \mathrm{m}$, the spin coater was set to ramp to $500 \mathrm{rpm}$ at $100 \mathrm{rpm} / \mathrm{sec}$, held for 5-10 seconds, and was ramped to a final spin speed of $3000 \mathrm{rpm}$ at $300 \mathrm{rpm} / \mathrm{sec}$, held for 30 seconds at that speed, and stopped gradually. Upon completion of this process, the wafer was soft baked in two steps. First, the wafer was pre-baked for 2 minutes at $65 \mathrm{C}$ and then soft-baked at $95 \mathrm{C}$ for 5 minutes. The next fabrication step was UV exposure. The total energy dose is $100-150 \mathrm{~mJ} / \mathrm{cm}^{2}$. On completion of exposure, the second step was to post-bake the wafer. During post bake, the wafer was baked at $65 \mathrm{C}$ for 1 minute then shifted to another hot plate to be baked at $95 \mathrm{C}$ for 2 minutes. Once the wafer was cooled, an SU-8 developer was used to wash away regions of unexposed SU-8 from the wafer and leave only the microstructures patterned on the surface. The wafer was submerged in a container with SU-8 10 developer for approximately two minutes. The container was gently agitated to allow complete removal of unexposed SU8 10. Isopropyl alcohol was then applied to wash away any developer left on the surface of the wafer. This wafer was once again rinsed with deionized water to remove any toxins that were present on the wafer. The wafer was then blow dried with a jet of Nitrogen gas, and the SU-8 pattern was ready for blotting and then extraction.

\section{Micromanipulation}

By micromanipulation we mean a procedure by which microstructures blotted with bacteria are extracted from the substrate and released into the working fluid with the aid of the microscope.

After the fabrication process, the two inch wafer with microstructures was cut into sections $10 \mathrm{~mm} \times 5 \mathrm{~mm}$ using a diamond tipped engraving pen, which contained several fully intact microstructures. To blot, the separated sections were washed with motility buffer $(0.01 \mathrm{M}$ potassium phosphate, $0.067 \mathrm{M}$ sodium chloride, $10^{-4} \mathrm{M}$ ethylenediaminetetraacetic acid (EDTA), $0.01 \mathrm{M}$ glucose, and $0.002 \%$ Tween-20, $\mathrm{pH}$ 7.0) then inverted onto the edge of the swarm plate. The section was removed from the swarm plate, transferred to a dish with motility buffer, and lightly agitated to remove unattached bacteria and excess agar. This process ensured that a monolayer of bacteria was attached to the microstructures with flagella free to move and untangled from other layers of bacteria and agar. The blotted section was then moved to a fresh Petri dish and submerged under a thin layer of motility buffer. The manipulation was performed using a stereo microscope for three-dimensional viewing, thus allowing individual microstructures to be selected and removed. After affixing the silicon chip to the bottom of the Petri dish, a 25 gauge needle was used to select and remove structures along the longest side.

\section{Microstructure tracking}

A tracking algorithm was designed to analyze the motion of the SU-8 microstructure driven by the attached flagellated 


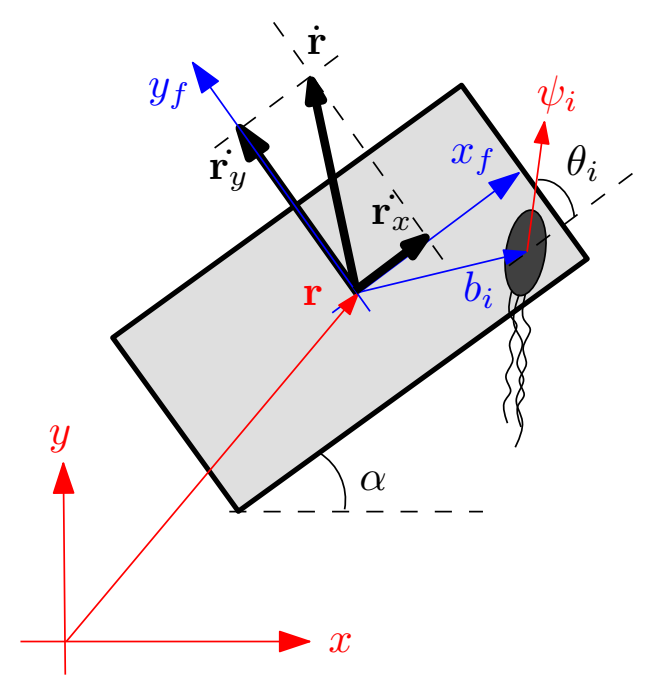

Fig. 2. A schematic of a microbarge and a bacterium. The angle $\alpha$ is formed by the main axis of the microbarge and the $x$ axis. The vector $\mathbf{r}$ denotes the position of the microbarge's center of mass. The vector $b_{i}$ denotes the position of the $i$-th bacterium w.r.t the microbarge's center of mass. The vector $\psi_{i}$ is a unit vector that denotes the orientation of the $i$-th bacterium. The angle $\theta_{i}$ is formed by the microbarges main axis and the orientation of the $i$-th bacterium.

bacteria $S$. marcescens in motility buffer. The current study analyzed two distinct motions of rigid bodies, translation and rotation. To characterize the motion of the bacteria-driven microstructures, the geometric centroid and orientation angle were traced. The algorithm was validated by testing the motion and velocity of a theoretical test structure with predetermined shape and velocity.

A set of consecutive frames with $2048 \times 2048$ pixels were captured using a Retiga 4000R digital camera and imported into MATLAB for analysis. The grayscale images were converted to binary images using a threshold tuned to optimize the effect of edge contrast of the SU-8 microstructure. The binary images were then inverted and all closed regions were filled. Closed structures of all sizes were next identified as individual elements, and elements smaller and larger than a predetermined pixel count were deleted leaving the area of the microstructure clearly defined and isolated. Finally, microstructure centroid location and orientation for each frame were determined.

\section{MATHEMATICAL MODELING AND ANALYSIS}

\section{A. Stochastic kinematic model}

The state of the microbarge is characterized by its position on the plane and its orientation. See Figure 2 for an illustration. We define the vector $\mathbf{r}=(x, y)$ to be the planar position of the microbarge's center of mass. The orientation of the microbarge is characterized by the angle $\alpha$, which is formed by the main axis of the microbarge and the $x$-axis of the inertial coordinate frame.

We assume that there are $N_{b}$ bacteria attached to a microbarge. The position of the $i$-th bacterium with respect to the center of mass of the microbarge is denoted by the vector $\mathbf{b}_{i}=\left(b_{i, x}, b_{i, y}\right)$ in the body-fixed coordinate frame, and its orientation is characterized by the angle $\theta_{i}$. We also define

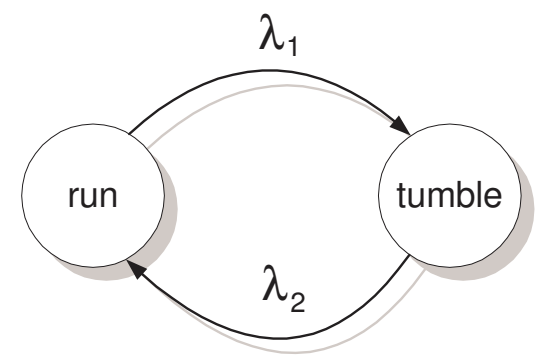

Fig. 3. A two-state continuous Markov chain model for the stochastic behavior of the bacteria. The transition rates between the states are given as $\lambda_{1}$ and $\lambda_{2}$. In chemical attractant free environment, measurements in biological experiments reveal that $\lambda_{1}=1 \mathrm{~s}^{-1}$ and $\lambda_{2}=10 \mathrm{~s}^{-1}$.

the amount of (time varying) propulsive force provided by the $i$-th bacterium as $p_{i}(t)$.

The equation of translational motion of the microbarge is given by

$$
M \frac{d^{2} \mathbf{r}}{d t^{2}}=\sum_{i=1}^{N_{b}} p_{i} \psi_{i}-k_{T} \frac{d \mathbf{r}}{d t}
$$

where $M$ is the total mass of the microbarge system (including the bacteria), $\psi_{i}$ is the unit vector in the inertial coordinate frame that represents the orientation of the $i$-th bacterium, and $k_{T}$ is the translational viscous drag coefficient. Similarly, the rotational motion can be characterized by

$$
I \frac{d^{2} \alpha}{d t^{2}}=\sum_{i=1}^{N_{b}} p_{i} \cdot\left(b_{i, x} \sin \theta_{i}-b_{i, y} \cos \theta_{i}\right)-k_{R} \frac{d \alpha}{d t},
$$

where $I$ is the total moment of inertia of the microbarge system and $k_{R}$ is the rotational viscous drag coefficient. In an environment with very low Reynolds number, the inertia effect is negligible, i.e. $k_{T} \gg M, k_{R} \gg I$.

Consequently, the translational and the rotational accelerations are negligible. Therefore, (1) and (2) can be accurately replaced with

$$
\begin{aligned}
\frac{d \mathbf{r}}{d t} & =\frac{1}{k_{T}} \sum_{i=1}^{N_{b}} p_{i} \psi_{i}, \\
\omega & :=\frac{d \alpha}{d t}=\frac{1}{k_{R}} \sum_{i=1}^{N_{b}} p_{i} \cdot\left(b_{i, x} \sin \theta_{i}-b_{i, y} \cos \theta_{i}\right) .
\end{aligned}
$$

The propulsion forces, $p_{i}(t)$, are stochastic processes. Biological investigation by Berg et al [13] reveals that in the absence of chemotactic chemical agents, the process can be accurately modeled as a continuous-time Markov chain [14] with two states, run and tumble (see Figure 3). We assume that during tumble, a bacterium does not provide any propulsion, while during run it delivers the maximal propulsive force of $p_{\max }=0.45 \mathrm{pN}$ reported in the literature [15].

If we define $\phi(t)=\left(\phi_{1}(t), \phi_{2}(t)\right)^{T}$ as the probability of finding the system in the run and tumbling state at time $t$, the evolution of $\phi(t)$ is given by

$$
\frac{d}{d t}\left[\begin{array}{l}
\phi_{1} \\
\phi_{2}
\end{array}\right]=\left[\begin{array}{cc}
-\lambda_{1} & \lambda_{2} \\
\lambda_{1} & -\lambda_{2}
\end{array}\right]\left[\begin{array}{l}
\phi_{1} \\
\phi_{2}
\end{array}\right] \text {. }
$$


From here, it follows that any initial distribution $\phi(0)$ converges exponentially to a steady state distribution given by

$$
\left[\begin{array}{l}
\phi_{1}(\infty) \\
\phi_{2}(\infty)
\end{array}\right]=\left[\begin{array}{c}
\frac{\lambda_{2}}{\lambda_{1}+\lambda_{2}} \\
\frac{\lambda_{1}}{\lambda_{1}+\lambda_{2}}
\end{array}\right]
$$

\section{B. Quantitative analysis of the microbarge rotation}

If we denote the parameter

$$
c_{i}:=\frac{b_{i, x} \sin \theta_{i}-b_{i, y} \cos \theta_{i}}{k_{R}},
$$

then the orientation of the barge $\alpha$ satisfies the following relation

$$
\alpha(t)=a(0)+\int_{0}^{t} \sum_{i=1}^{N_{b}} c_{i} \cdot p_{i}(\tau) d \tau .
$$

From here, we can compute the expectation of $\alpha(t)$ as

$$
E(\alpha(t))=\alpha(0)+\bar{p} \sum_{i=1}^{N_{b}} c_{i} t .
$$

Here we use the assumption that at the beginning of the time interval of interest, $t=0$, the processes $p_{i}(t)_{i=1 \ldots N_{b}}$ have reached their steady state. In that case, their expectation is then given by the steady state expected value, $\bar{p}$, which can be computed as

$$
\bar{p}=\frac{\lambda_{2}}{\lambda_{1}+\lambda_{2}} \cdot p_{\max }=0.41 \mathrm{pN} .
$$

Similarly, we can compute the variance of $\alpha(t)$ as follows.

$$
\begin{gathered}
\operatorname{Var}(\alpha(t))=E\left(\int_{0}^{t} \sum_{i=1}^{N_{b}} c_{i} \cdot\left(p_{i}(\tau)-\bar{p}\right) d \tau\right)^{2}, \\
=E\left(\int_{0}^{t} \int_{0}^{t} \sum_{i=1}^{N_{b}} c_{i} \cdot\left(p_{i}(\tau)-\bar{p}\right) \sum_{j=1}^{N_{b}} c_{j} \cdot\left(p_{j}(\eta)-\bar{p}\right) d \tau d \eta\right), \\
=\int_{0}^{t} \int_{0}^{t} \sum_{i=1}^{N_{b}} \sum_{j=1}^{N_{b}} c_{i} \cdot c_{j} \cdot\left(E\left(p_{i}(\tau) p_{j}(\eta)\right)-\bar{p}^{2}\right) d \tau d \eta . \quad(10)
\end{gathered}
$$

Assuming that the random behavior of the bacteria are independent one from another, we can simplify (10) into

$$
\operatorname{Var}(\alpha(t))=2 \int_{0}^{t} \int_{\eta}^{t} \sum_{i=1}^{N_{b}} c_{i}^{2} \cdot\left(E\left(p_{i}(\tau) p_{i}(\eta)\right)-\bar{p}^{2}\right) d \tau d \eta
$$

Furthermore, using the above mentioned assumption that the processes have reached the steady state at $t=0$, we can compute $E\left(p_{i}(\tau) p_{i}(\eta)\right)$ through the Bayesian formula. The values of $P\left\{\left(p_{i}(\tau)=A\right),\left(p_{i}(\eta)=B\right)\right\}$ is given in the following table.

\begin{tabular}{|c|cc|}
\hline$A \backslash B$ & $p_{\max }$ & 0 \\
\hline$p_{\max }$ & $\frac{\lambda_{2}^{2}+\lambda_{1} \lambda_{2} e^{\left(\lambda_{1}+\lambda_{2}\right)(\eta-\tau)}}{\left(\lambda_{1}+\lambda_{2}\right)^{2}}$ & $\frac{\lambda_{1} \lambda_{2}-\lambda_{1} \lambda_{2} e^{\left(\lambda_{1}+\lambda_{2}\right)(\eta-\tau)}}{\left(\lambda_{1}+\lambda_{2}\right)^{2}}$ \\
0 & $\frac{\lambda_{1} \lambda_{2}-\lambda_{1} \lambda_{2} e^{\left(\lambda_{1}+\lambda_{2}\right)(\eta-\tau)}}{\left(\lambda_{1}+\lambda_{2}\right)^{2}}$ & $\frac{\lambda_{1}^{2}+\lambda_{1} \lambda_{2} e^{\left(\lambda_{1}+\lambda_{2}\right)(\eta-\tau)}}{\left(\lambda_{1}+\lambda_{2}\right)^{2}}$ \\
\hline
\end{tabular}

We can compute that

$$
E\left(p_{i}(\tau) p_{i}(\eta)\right)=\frac{\lambda_{2}^{2}+\lambda_{1} \lambda_{2} e^{\left(\lambda_{1}+\lambda_{2}\right)(\eta-\tau)}}{\left(\lambda_{1}+\lambda_{2}\right)^{2}} p_{\max }^{2},
$$

and

$$
\operatorname{Var}(\alpha(t))=\frac{2 \lambda_{1} \lambda_{2} p_{\max }^{2}}{\left(\lambda_{1}+\lambda_{2}\right)^{3}} \sum_{i=1}^{N_{b}} c_{i}^{2} \cdot\left(t-\frac{1-e^{-\left(\lambda_{1}+\lambda_{2}\right) t}}{\lambda_{1}+\lambda_{2}}\right) .
$$

From (13), we see that both the expectation and the variance of $\alpha(t)$ grow asymptotically linearly. The standard deviation of $\alpha(t)$ grows asymptotically with $\sqrt{t}$, which is half an order slower than the expectation. Consequently, as $t \rightarrow \infty$, the ratio of the standard deviation to the expectation goes to 0 . This means the expectation can be used as a good estimate of the steady state behavior of the system. The expectation of $\alpha(t)$ predicts that the microbarge undergoes a steady rotation as a steady state behavior. In the next section, we will see that this is justified by the experimental results (see Figure 5(a)).

Notice that the assumption that the random behavior of the bacteria are independent one from another is not essential in deriving this result. To see this, consider the extreme case, where all the bacteria are perfectly correlated. In this case, the term $\sum_{i=1}^{N_{b}} c_{i}^{2}$ in (13) will be replaced by $\sum \sum_{i, j=1}^{N_{b}} c_{i} c_{j}$, which does not change the conclusion.

\section{PARAMETER ESTIMATION AND MODEL VALIDATION}

The components of the translational velocities on the axis of the body fixed coordinate frame (see Figure 2) are

$$
v_{x}:=\dot{r}_{x}=\frac{1}{k_{T}} \sum_{i=1}^{N_{b}} p_{i} \cos \theta_{i}, v_{y}:=\dot{r}_{y}=\frac{1}{k_{T}} \sum_{i=1}^{N_{b}} p_{i} \sin \theta_{i} .
$$

Their respective expectations are then given by

$$
E v_{x}=\frac{\bar{p}}{k_{T}} \sum_{i=1}^{N_{b}} \cos \theta_{i}, E v_{y}=\frac{\bar{p}}{k_{T}} \sum_{i=1}^{N_{b}} \sin \theta_{i} .
$$

From (3), we can obtain the expectation of the angular velocity of the microbarge, which is given by

$$
E \omega=\frac{\bar{p}}{k_{R}} \sum_{i=1}^{N_{b}}\left(b_{i, x} \sin \theta_{i}-b_{i, y} \cos \theta_{i}\right) .
$$

It is clear from (14-16) that the expected velocities only depend on three parameters:

$$
\begin{aligned}
& \beta_{1}:=\frac{1}{k_{T}} \sum_{i=1}^{N_{b}} \cos \theta_{i}, \beta_{2}:=\frac{1}{k_{T}} \sum_{i=1}^{N_{b}} \sin \theta_{i}, \\
& \beta_{3}:=\frac{1}{k_{R}} \sum_{i=1}^{N_{b}}\left(b_{i, x} \sin \theta_{i}-b_{i, y} \cos \theta_{i}\right) .
\end{aligned}
$$

\section{A. Parameter estimation}

We estimate the values of these parameters using experimental data. We extract frames from the video taken during the experiment. In each frame, the position and orientation of the barge are identified using digital image processing. As the results, we have three time series $\left\{\bar{x}_{i}\right\},\left\{\bar{y}_{i}\right\}$, and $\left\{\bar{\alpha}_{i}\right\}$, with 

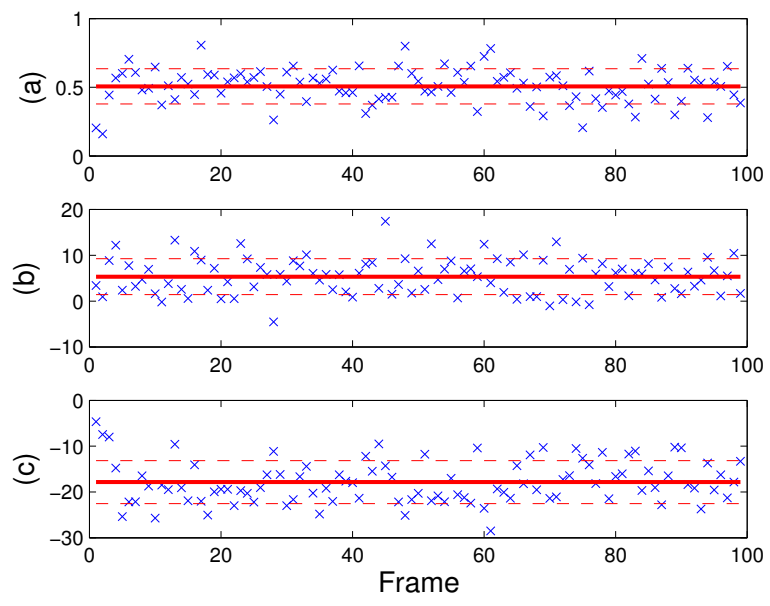

Fig. 4. The computed data for a rectangular microbarge $(50 \mu \mathrm{m} \times 100 \mu \mathrm{m})$. (a) $\left\{\bar{\omega}_{i}\right\}$ in rad/s, (b) $\left\{\bar{v}_{x, i}\right\}$ in $\mu \mathrm{m} / \mathrm{s}$, (c) $\left\{\bar{v}_{y, i}\right\}$ in $\mu \mathrm{m} / \mathrm{s}$. The solid lines show the averages of the data, while the gaps between the solid lines and the dashed lines represent the standard deviations.

$i=1, \ldots, N$, consisting of the planar position of the barge and its orientation in $N$ frames. The body fixed coordinate components of the microbarge's translational velocity at the $i$-th frame can be approximated by using the forward difference method as follows.

$$
\left[\begin{array}{c}
\bar{v}_{x, i} \\
\bar{v}_{y, i}
\end{array}\right]=\frac{1}{\delta}\left[\begin{array}{cc}
\cos \bar{\alpha}_{i} & \sin \bar{\alpha}_{i} \\
-\sin \bar{\alpha}_{i} & \cos \bar{\alpha}_{i}
\end{array}\right]\left[\begin{array}{c}
\bar{x}_{i+1}-\bar{x}_{i} \\
\bar{y}_{i+1}-\bar{y}_{i}
\end{array}\right],
$$

for $i \in\{1, \ldots, N-1\}$, where $\delta$ is the video sampling rate. Similarly, the angular velocity of the microbarge can be extracted from the video data by $\bar{\omega}_{i}=\frac{\bar{\alpha}_{i+1}-\bar{\alpha}_{i}}{\delta}$.

By equating the averages and the expectations of the microbarge's translational and angular velocities, we can estimate the values of $\beta_{1,2,3}$ as follows.

$$
\left[\begin{array}{lll}
\beta_{1} & \beta_{2} & \beta_{3}
\end{array}\right] \approx \frac{1}{\bar{p}(N-1)} \sum_{i=1}^{N-1}\left[\begin{array}{ccc}
\bar{v}_{x, i} & \bar{v}_{y, i} & \bar{\omega}_{i}
\end{array}\right] .
$$

Figure 4 shows the computed $\left\{\bar{\omega}_{i}\right\},\left\{\bar{v}_{x, i}\right\}$, and $\left\{\bar{v}_{y, i}\right\}$ for a rectangular microbarge $(50 \mu \mathrm{m} \times 100 \mu \mathrm{m})$ as shown in Figure 1. The video length is 10 seconds, sampled at 10 frames/second. Based on this data, the parameters for this microbarge are computed as $\beta_{1}=13.03 \frac{\mu \mathrm{m}}{\mathrm{s} \mathrm{pN}}, \beta_{2}=$ $-43.64 \frac{\mu \mathrm{m}}{\mathrm{s} \mathrm{pN}}$, and $\beta_{3}=1.24 \frac{\mathrm{rad}}{\mathrm{s} \mathrm{pN}}$.

The three parameters $\beta_{1,2,3}$ summarize the distribution of the bacteria on the microbarge. Subsequently, we will show that our mathematical model and the parameters $\beta_{1,2,3}$ can predict the behavior of the system reasonably well.

\section{B. Model validation}

In this subsection, we show that the mathematical model developed in the previous section and the parameters $\beta_{1,2,3}$ can predict the behavior of the system reasonably well. We construct a deterministic model by replacing the stochastic processes $p_{i}(t)$ in (3) with their steady state expectations $\bar{p}$. We therefore construct a reduced-order model for the system, which is given by $\dot{x}=\bar{p}\left(\beta_{1} \cos \alpha-\beta_{2} \sin \alpha\right)$, $\dot{y}=\bar{p}\left(\beta_{1} \sin \alpha+\beta_{2} \cos \alpha\right)$, and $\dot{\alpha}=\bar{p} \beta_{3}$.
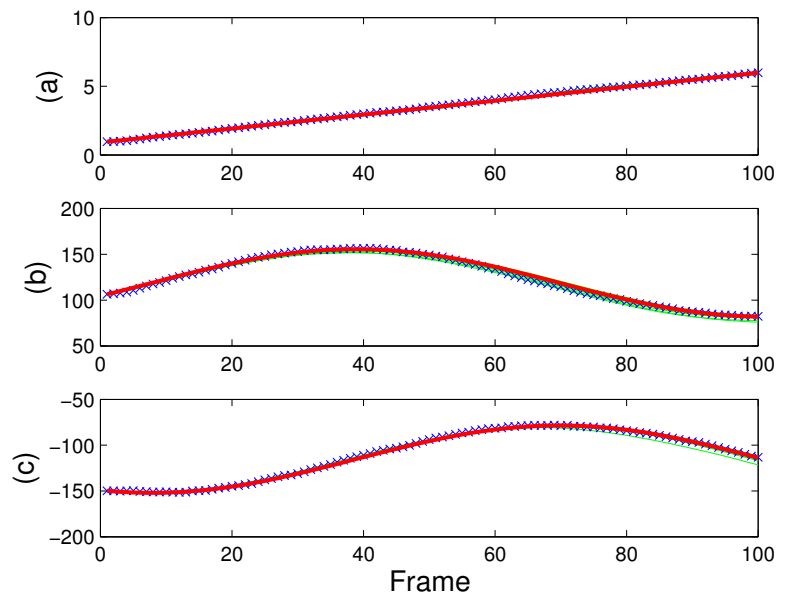

Fig. 5. The comparison between the experimental data (x), the deterministic model prediction (thick line), and stochastic simulations (solid lines) for a rectangular microbarge $(50 \mu \mathrm{m} \times 100 \mu \mathrm{m})$. (a) $\alpha$ in $\mathrm{rad} / \mathrm{s}$, (b) $x$ in $\mu \mathrm{m}$, (c) $y$ in $\mu \mathrm{m}$.

Figure 5 shows the comparison between the experimental data, the deterministic model prediction and the stochastic simulations of the model (3) for the rectangular microbarge that is analyzed in the previous section. Note that for each simulation run, the distribution of 300 bacteria on the microbarge is randomized while keeping the parameters $\beta_{1,2,3}$ constant.

We can see that the model with fitted parameter can explain the data very well, suggesting that the structure of the model is suitable for this experimental setup. Furthermore, we can observe that the distributed parameter model that includes the description of the distribution of the bacteria on the microbarge $\left(\mathbf{r}_{i}\right.$ and $\left.\theta_{i}\right)$ can be replaced with a lumped parameter model with the initial state of the system and three parameters of bacterial distribution $\left(\beta_{1,2,3}\right)$. Therefore, in order to describe the dynamics of the system accurately, it is not necessary to know how the bacteria are distributed precisely. Rather, it is sufficient to know a few high level parameters that describe the distribution.

\section{The effect of orientation coherence on microbarge actu- ation}

Due to the nature of the blotting process, the distribution of the bacteria on the microbarge (both position and orientation) is inherently random. In this subsection, we analyze the effect of coherence in the orientation distribution and the kinematic behavior of the microbarge.

Consider the expectation of the magnitude of the translational velocity of the microbarge, as given in (3a).

$$
E\left\|\frac{d \mathbf{r}}{d t}\right\|=\frac{\bar{p}}{k_{T}}\left\|\sum_{i=1}^{N_{b}} \psi_{i}\right\| .
$$

Since this quantity does not depend on the choice of coordinate frame, we can conveniently evaluate it in the body fixed 

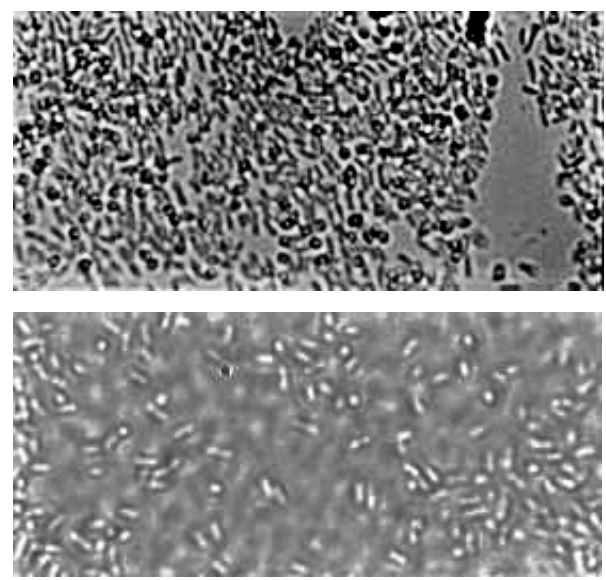

Fig. 6. Microscopy image of Microbarge A (top) and Microbarge B (bottom) and the bacteria on their surface.
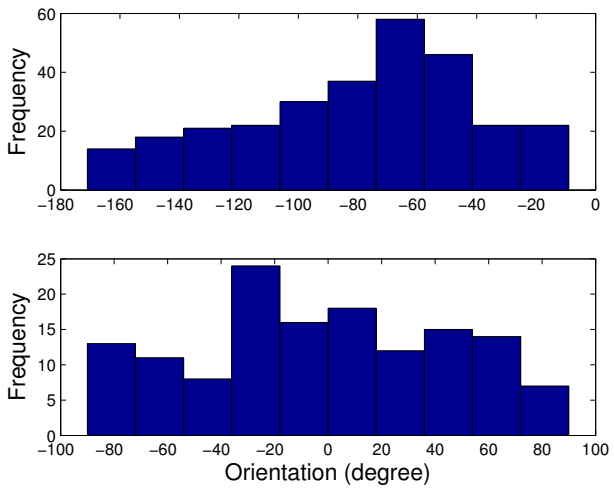

Fig. 7. Histograms of the orientation of the bacteria on Microbarge A (top) and Microbarge B(bottom).

coordinate frame. In this case, the right hand side becomes

$$
\frac{\bar{p}}{k_{T}}\left\|\sum_{i=1}^{N_{b}} \psi_{i}\right\|=\frac{\bar{p} N_{b}}{k_{T}}\left(\frac{1}{N_{b}^{2}} \sum_{i=1}^{N_{b}} \sum_{j=1}^{N_{b}} \cos \left(\theta_{i}-\theta_{j}\right)\right)^{\frac{1}{2}} \text {. }
$$

The quantity between the brackets in (19) can be seen as a measure of the coherence of the orientation of bacteria. If all of them have the same orientation, this quantity is 1 . If there is no correlation between the orientation, the expected value of the cosine function is zero, and so is this quantity. We can therefore propose a measure of orientation coherence, based on experimental data, as $\gamma:=v_{\text {avg }} k_{T} /\left(\bar{p} N_{b}\right)$. By comparing measurements from two different microbarges, we can justify this idea.

Figure 6 shows microscopy images of two microbarges. From these two images, we can extract some information about the distribution of the bacteria on their surface. Through digital image processing, we can extract the information about the alignment of the major axis of the bacteria on both microbarges. The statistics of this data is shown in Figure 7.

We can compute $\gamma$ for both microbarges by using the recorded average velocities and the number of identified bacteria on each microbarge. The $\gamma$ values are $\gamma_{A}=0.157 k_{T}$ and $\gamma_{B}=0.5154 k_{T}$. Visual inspection on the histograms shown in Figure 7 does not reveal too much information about the orientation coherence in both microbarges. However, we can approximately ${ }^{2}$ compute the term between brackets in (19) for both barges, which are 0.37 and 0.49 for Microbarge A and B, respectively. Therefore, it is likely that the bacteria are more coherently oriented on Microbarge B than on Microbarge A.

\section{CONCLUSION}

We developed a model of a microstructure blotted with bacteria moving in a micro channel propelled by the flagella of the bacteria. The main contribution of the paper is the reduction of the complex dynamics of the system of bacteria and the microstructure to a system of three ordinary differential equations with only three parameters. The predictions from the reduced-order model are consistent with the experimental data for the motion of the microstructure. Our future work addresses the feedback control of the microstructures using phototactic, galvanotactic and chemotactic inputs using such reduced-dimensional models to steer the system through micro channels.

\section{REFERENCES}

[1] J. Fang, K. Wang, and K. F. Böhringer, "Self-assembly of PZT actuators for micro pumps with high process repeatability," ASME/IEEE Journal of Microelectromechancial Systems, vol. 15, no. 4, pp. 871878, 2006.

[2] J. J. Abbott, Z. Nagy, F. Beyeller, and B. J. Nelson, "Robotics in the small," IEEE Robotics and Automation Magazine, pp. 92-103, June 2007.

[3] D. B. Weibel, P. Garstecki, D. Ryan, W. R. DiLuzio, M. Mayer, J. E. Seto, and G. M. Whitesides, "Microoxen: Microorganisms to move microscale loads," Proc. National Academy of Science, vol. 102, no. 34, pp. 11963-11967, 2005.

[4] D. J. Bell, S. Leutenegger, K. M. Hammar, L. X. Dong, and B. J. Nelson, "Flagella-like propulsion for microrobots using a nanocoil and a rotating electromagnetic field," in Proc. IEEE Int. Conf. Robotics and Automation, (Rome, Italy.), pp. 1128 - 1133, 2007.

[5] S. Martel, C. Tremblay, S. Ngakeng, and G. Langlois, "Controlled manipulation and actuation of micro-objects with magnetotactic bacteria," Applied Physics Letters, vol. 89, p. 233904, December 2006.

[6] B. Behkam and M. Sitti, "Bacterial flagella-based propulsion and on/off motion control of microscale objects," Applied Physics Letters, vol. 90, no. 2, pp. 1-3, 2007.

[7] E. Steager, C.-B. Kim, C. Naik, J. Patel, S. Bith, L. Reber, and M. J. Kim, "Control of microfabricated structures powered by flagellated bacteria using phototaxis," Applied Physics Letters, vol. 90, no. 26, p. 263901, 2007.

[8] H. C. Berg and D. A. Brown, "Chemotaxis in Escherichia coli analysed by three-dimensional tracking," Nature, vol. 239, no. 5374, pp. 500-504, 1972.

[9] H. C. Berg, E. coli in motion. New York: Springer-Verlag, 2004.

[10] H. C. Berg and R. A. Anderson, "Bacteria swim by rotating their flagellar filaments," Nature, vol. 245, no. 5425, pp. 380-382, 1973

[11] B. Behkam and M. Sitti, "Effect of quantity and configuration of attached bacteria on bacterial propulsion of microbeads," Applied Physics Letters, vol. 93, p. 223901, 2008.

[12] J. Ueda, L. Odhner, and H. Asada, "Broadcast feedback of stochastic cellular actuators inspired by biological muscle control," Int. Journal of Robotics Research, vol. 26, no. 11, pp. 1251-1266, 2007.

[13] H. C. Berg, "Motile behavior of bacteria," Physics Today, vol. 53, no. 1 , pp. $24-29,2000$

[14] C. G. Cassandras and S. Lafortune, Introduction to Discrete Event Systems. Kluwer, 1999.

[15] N. C. Darnton, L. Turner, S. Rojevsky, and H. C. Berg, "On torque and tumbling in swimming Escherichia coli," Journal of Bacteriology, vol. 189 , no. 5 , pp. 1756-1764, 2007.

\footnotetext{
${ }^{2}$ Not all bacteria can be successfully identified by the digital image processing algorithm. Moreover, the identified orientation is that of the major axis of the bacteria, instead of the bacteria themselves.
} 